\title{
Introducing the DizzyQuest: an app-based diary for vestibular disorders
}

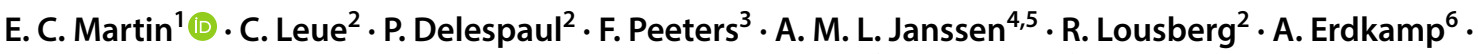 \\ S. van de Weijer ${ }^{6} \cdot$ J. Widdershoven ${ }^{1} \cdot$ H. Blom ${ }^{7} \cdot$ T. Bruintjes $^{8} \cdot$ A. Zwergal ${ }^{9} \cdot$ E. Grill $^{10} \cdot$ N. Guinand ${ }^{11}$. \\ A. Perez-Fornos ${ }^{11} \cdot$ M. R. van de Berg ${ }^{1} \cdot$ J. J. A. Stultiens ${ }^{1} \cdot$ H. Kingma ${ }^{1,12} \cdot$ R. van de Berg ${ }^{1,12}$
}

Received: 27 March 2020 / Revised: 8 July 2020 / Accepted: 16 July 2020 / Published online: 25 July 2020

(c) The Author(s) 2020

\begin{abstract}
Background Most questionnaires currently used for assessing symptomatology of vestibular disorders are retrospective, inducing recall bias and lowering ecological validity. An app-based diary, administered multiple times in daily life, could increase the accuracy and ecological validity of symptom measurement. The objective of this study was to introduce a new experience sampling method (ESM) based vestibular diary app (DizzyQuest), evaluate response rates, and to provide examples of DizzyQuest outcome measures which can be used in future research.

Methods Sixty-three patients diagnosed with a vestibular disorder were included. The DizzyQuest consisted of four questionnaires. The morning- and evening-questionnaires were administered once each day, the within-day-questionnaire 10 times a day using a semi-random time schedule, and the attack questionnaire could be completed after the occurrence of a vertigo or dizziness attack. Data were collected for 4 weeks. Response rates and loss-to-follow-up were determined. Reported symptoms in the within-day-questionnaire were compared within and between patients and subgroups of patients with different vestibular disorders.

Results Fifty-one patients completed the study period. Average response rates were significantly higher than the desired response rate of $>50 \%(p<0.001)$. The attack-questionnaire was used 159 times. A variety of neuro-otological symptoms and different disease profiles were demonstrated between patients and subgroups of patients with different vestibular disorders. Conclusion The DizzyQuest is able to capture vestibular symptoms within their psychosocial context in daily life, with little recall bias and high ecological validity. The DizzyQuest reached the desired response rates and showed different disease profiles between subgroups of patients with different vestibular disorders. This is the first time ESM was used to assess daily symptoms and quality of life in vestibular disorders, showing that it might be a useful tool in this population.
\end{abstract}

Keywords DizzyQuest $\cdot$ Experience sampling $\cdot$ Vestibular disorders

\section{Introduction}

Dizziness, imbalance and vertigo are common complaints, with a prevalence of up to $36 \%$ in the outpatient population [1]. A subset of these complaints is caused by vestibular disorders [2]. These vestibular disorders can present with different clinical pictures, varying from acute attacks of vertigo

Electronic supplementary material The online version of this article (https://doi.org/10.1007/s00415-020-10092-2) contains supplementary material, which is available to authorized users.

R. van de Berg

raymond.vande.berg@mumc.nl

Extended author information available on the last page of the article to chronic complaints [3-5]. Patients with a vestibular disorder can also suffer from other problems like depression, increased risk of falling and cognitive impairment, with many socio-economic consequences [6]. To evaluate the symptoms and consequences of vestibular disorders, questionnaires like the Dizziness Handicap Inventory (DHI) [7], the Hospital Anxiety and Depression Scale (HADS) [8], the Short-Form Health Survey (SF-36) [9], Short Falls Efficacy Scale-International (Short FES-I) [10], and the EQ-5D-5L are often used.

However, there are two main drawbacks tied to the use of cross-sectional instruments. First, most of these questionnaires are retrospective, and require recollection and adequate aggregation. This imposes a high risk of recall 
bias, since some variables might be overestimated or underestimated. Secondly, most of these questionnaires only yield one observation and miss documenting variability in symptomatology or risk patterns in the context of daily life. Cross-sectional instruments are not able to capture dynamic components (e.g., fluctuating symptoms) and the influence of context (e.g., environmental or psychological factors, etc.), which are relevant indicators for symptom management. Understanding symptoms in the normal flow of daily life improves the ecological validity: it better reflects the real life response of symptoms to real life challenges [11-13]. Especially in vestibular disorders which can have a very fluctuating clinical presentation with vertigo attacks, questionnaires prone to recall bias and lower ecological validity, are suboptimal to study the exact frequency of attacks, their possible triggers and the quality of life in vestibular disorders.

In the past, the Experience Sampling Method (ESM) was developed to overcome recall bias and to increase the ecological validity of measurements of health outcomes. Historically, paper and pencil procedures were burdensome. Recently, apps on a smartphone improved data collection logistics. In ESM the information about an individuals' current state and functioning, as well as circumstances and context, is documented by self-report in real-time using a random time sampling procedure to repeatedly fill in questionnaires for a longer period of time. The questionnaires have to be filled out multiple times a day, at most 10 times. On these moments, a notification beep alerts the subject to fill out an assessment form. A questionnaire can also be filled out by the subject in case of a certain trigger or event [11]. ESM not only reduces the risk of recall bias, it also makes it possible to obtain information about the variability of attacks over time [13]. Furthermore, the existence and influence of environmental, social and psychological factors can be investigated at the same time [14]. Momentary assessment has already been successfully used in studies on other diseases, e.g., tinnitus [15], psychological disorders [16-18] and Parkinson's disease [19]. However, there is only scarce evidence in vestibular disorders, using techniques that to a certain extent resemble ESM [14].

Since patients often bring their own (non-standardized) "vestibular diary" to the clinic and currently many new therapeutic strategies are being investigated [20-40], standardized monitoring of patients using ESM improves clinical work and research, complementary to existing questionnaires. Therefore, the Dizzynet network [41, 42] developed a new app-based diary for vestibular disorders: the DizzyQuest. Main objective of this study was to describe the first version of the DizzyQuest, to evaluate response rates, and to provide examples of DizzyQuest outcome measures which can be used in future research, like the variability of neurootological symptoms over time using the ESM method, and the relationships between symptoms and their psychosocial context.

\section{Methods}

\section{Study participants}

Patients with various vestibular disorders were recruited at the vestibular department of Maastricht University Medical Center, Apeldoorn Dizziness Center, Haga Hospital and the 'Hoormij' foundation, all in the Netherlands. Inclusion criteria comprised: (1) age 18 years or older; (2) diagnosed with a vestibular disorder by an otorhinolaryngologist with an expertise in vestibular disorders, according to the Barany Society diagnostic criteria [43-46] (if applicable); (3) good understanding of the Dutch language (reading and listening); (4) in possession of a smartphone or tablet; (5) being capable to install and use the DizzyQuest (see below for explanation of the application). Patients were excluded if they did not feel comfortable to answer questions from the DizzyQuest, for example, concerning their psychological status.

\section{The DizzyQuest}

The DizzyQuest is an app-based electronic diary that is implemented on the "UM ESM" (v2.04) platform. This is an experimental version of the PsyMate ${ }^{\mathrm{TM}}$ app (http://www. psymate.eu) used for research at Maastricht University. The app allows implementation of ESM based protocols with multiple questionnaires and sampling schemes [47]. It is available as a download for smartphones running on iOS and Android. The DizzyQuest consists of four questionnaires. The "within-day-questionnaire" assesses the current situation at semi-random intervals throughout the day in the tradition of the ESM method for 10 times a day. The other three questionnaires are retrospective, in principle reflecting an aggregated situation of the previous night (morning questionnaire), or previous day (evening questionnaire). The attack questionnaire is event based and filled in after the occurrence of an attack of vertigo or dizziness (Table 1). It can be completed at any time after an attack, even after more than 1 day (Online Resource 1). Each questionnaire has its own specific set of questions, although considerable overlap exists between some of them (e.g. the within-day-questionnaire and the evening-questionnaire, see Online Resource 1 for the translated version). All four questionnaires combined, not only measure vestibular symptoms, but also emotion, cognition, context and stressful events. All questions about symptoms use the seven-point Likert scale, ranging from 1 (not at all) to 7 (very). It should be noted that the questionnaires were provided in Dutch. In this language, "dizziness" and "vertigo" are often used interchangeably in contrast to 
Table 1 Questionnaires used in the DizzyQuest

\begin{tabular}{llll}
\hline Questionnaire & Focus & Nature & Frequency in this study \\
\hline $\begin{array}{l}\text { Morning (7 questions) } \\
\text { Within-day (23 questions) }\end{array}$ & $\begin{array}{l}\text { Quality of sleep past night } \\
\text { Real-time symptoms in daily life } \\
\text { context }\end{array}$ & $\begin{array}{l}\text { Retrospective } \\
\text { Momentary (=ESM) }\end{array}$ & $\begin{array}{c}\text { Daily in first week } \\
\text { moments in the first week } \\
\text { Evening (27 questions) }\end{array}$ \\
$\begin{array}{l}\text { Symptoms of the past day } \\
\text { Attack (5 questions) }\end{array}$ & Symptoms of attack & $\begin{array}{c}\text { Retrospective } \\
\text { Retrospective }\end{array}$ & $\begin{array}{c}\text { Daily during 4 weeks } \\
\text { After an attack of vertigo or dizziness }\end{array}$ \\
\hline
\end{tabular}

Table 2 Possible outcome measures of the DizzyQuest (on individual level)

\begin{tabular}{ll}
\hline Outcome measure & $\begin{array}{l}\text { Facilitating } \\
\text { question- } \\
\text { naire }\end{array}$ \\
\hline $\begin{array}{l}\text { Behaviour of symptoms during the day } \\
\text { Behaviour of symptoms between days }\end{array}$ & $\begin{array}{l}\text { Within-day } \\
\text { Morning } \\
\text { Within-day }\end{array}$ \\
Appearance of neuro-otological symptoms in relation & $\begin{array}{l}\text { Evening } \\
\text { Morning }\end{array}$ \\
to psychosocial context & Within-day \\
Frequency and nature of attack of vertigo or dizziness & Evening \\
& Attack \\
& Evening \\
\hline
\end{tabular}

the English language, in which "dizziness" and "vertigo" are considered two different entities [48].

These different strategies (momentary versus retrospective) and different questionnaires, provide the opportunity to measure multiple aspects of neuro-otological symptoms and their psychosocial context. Table 2 provides possible outcome measures that can be obtained by the DizzyQuest on an individual level.

\section{Study design}

Before start of the DizzyQuest study, patients were contacted by two of the authors (SvdW, AE) and instructed on how to use the app. An online video was used for instructing the patients how to use the DizzyQuest (Online Resource 2). After that, the DizzyQuest was used for four sequential weeks. In this study, not all questionnaires were applied in the same frequency and for the same duration (Table 1). In the first week, each day contained one morning-questionnaire, 10 within-day-questionnaires and one eveningquestionnaire. Subjects received the within-day-questionnaires 10 times a day, between 07:30 a.m. and 22:30 p.m., at semi-random moments (a day consisted of ten 90-min blocks and at a random moment in each block, a questionnaire was sent to the patient). The morning-, day- and evening-questionnaires were activated at 04:00 a.m. and 07:30 p.m. respectively, with a reminder on the smartphone or tablet at 9:00 a.m. and 8:00 p.m. respectively. They could be answered for a period of 8 and $8.5 \mathrm{~h}$, respectively. The within-day-questionnaires were activated after a signalling beep/notification on the device. The subject was able to fill in the questionnaire within a time window of $15 \mathrm{~min}$. When the opportunity to fill in the questionnaire closed, the questionnaires disappeared from the home screen and data were archived as "negative response". The attack-questionnaire could be used at any given moment, as many times a day as needed. Subjects were instructed on the first day and debriefed on day 28. After the first week, only the evening- and attack-questionnaires remained. Therefore, in the last 3 weeks, patients only had to fill in one questionnaire a day (the evening-questionnaire), and optionally the attackquestionnaire in case of an attack of vertigo or dizziness, to reduce the burden for subjects.

\section{Patient monitoring}

The ESM UM Reporting Tool provides the opportunity for online monitoring of results. It was used to remotely monitor compliance of patients by two of the authors (SvdW, AE). In case a patient did not start the DizzyQuest at the predetermined day, or when sequentially multiple questionnaires were not answered, patients were contacted by phone or e-mail to provide any help if necessary (e.g., technical help).

\section{Data analysis}

Patients that started in the first week and continued the use of the DizzyQuest up to and including the fourth week, were included in the analysis. No minimum threshold of response rates was required. DizzyQuest data were extracted from the ESM UM database and transferred to IBM SPSS Statistics Version 25 (IBM Corporation, New York, United States of America). Data from the first day (briefing) was incomplete and skipped from the analysis, leaving only within-day-questionnaire data from day two until seven. The morning questionnaire was available from day two until eight, resulting in 7 days of data to be used for analysis. The evening- and attack-questionnaires were available for the whole 28 days period. Descriptive statistics were used. Furthermore, a Mann-Whitney U test, Fisher's Exact test 
and a Fisher-Freeman-Halton exact test of independence were used to calculate differences in age, gender and diagnosis respectively, between the study group and drop-out group. The average response rate of each patient for each questionnaire was calculated by dividing the amount of fully completed questionnaires by the number of scheduled questionnaires. Only fully completed questionnaires were considered as valid. It was hypothesized that not all administered questionnaires would be completed. A desired response rate of $>50 \%$ was used, since reported response rates in current literature about momentary assessments are often higher than $50 \%[49,50]$. To calculate whether response rates of the DizzyQuest questionnaires were significantly different from the $>50 \%$ desired response rate $[47,51]$, a one-sample $T$ test was performed.

Regarding presence of neuro-otological symptoms, they were considered "reported" and present, when they were selected in the question: 'Now I suffer from' (Online Resource 1). The reported frequency (\%) of neuro-otological symptoms obtained with the within-day questionnaire, was first calculated for each symptom per patient. This was done by dividing the amount of times a specific symptom was reported, by the amount of times the individual patient completed the within-day questionnaire. This resulted in a patient-specific percentage. This patient-specific percentage was used to determine the mean reported frequency (\%) of all patients, divided in three subgroups of vestibular disorders: Menière's disease (MD), bilateral vestibulopathy (BV), and other vestibular disorders.

Marginal logistic regression analysis based on the generalized estimated equations analysis (GEE) method for binary outcomes was used to test significant differences in reported symptoms between subgroups of vestibular disorders and the outcomes were Bonferroni corrected. An exchangeable covariance structure was applied, since time interval between within-day questionnaires was not constant.

To investigate the effect of neuro-otological symptoms on questions regarding positive affect and negative affect, linear mixed-effects regression analysis was performed. The independent variables in the models were one of the neuro-otological symptoms ("imbalance", "tinnitus" and "visual problems when moving"). The score on one of the questions regarding positive affect ("I generally feel well at the moment") and negative affect ("I'm worrying about things") was the dependent variable. In case of a significant interaction was found between the diagnosis group and the neuro-otological symptom, the analysis was performed for each diagnosis group separately.

To investigate differences between the three subgroups regarding amount of completed attack-questionnaires, the mean number of completed attack-questionnaires was first calculated per day for each patient by [amount of attack questionnaires completed by a specific patient/28 days].
Subsequently, a Kruskal-Wallis test was used to test for systematic differences between the three groups. In case of a significant Kruskal-Wallis test, post hoc analyses included three pairwise Bonferroni corrected Mann-Whitney U tests. For all performed statistic tests, $p$-values below 0.05 were considered statistically significant.

\section{Ethical considerations}

This study was in accordance with the legislation and ethical standards on human experimentation in the Netherlands and in accordance with the Declaration of Helsinki (amended version 2013). The medical ethical committee of Maastricht UMC+ approved this study (2018-0809) and written informed consent was obtained from all patients, prior to participation.

\section{Results}

\section{Patient characteristics and drop-out}

Sixty-three patients were included in this study. Twelve patients dropped out of the study for a variety of reasons (e.g., technical problems or personal circumstances) as described in Table 3. Fifty-one patients completed the 4-week study period. This group consisted mostly of females $(n=33)$ and had a mean age of 56 years old (range 27-80 years). Etiologies comprised Menière's disease $(n=19)$, vestibular migraine $(n=3)$, vestibulopathy $(n=1$ unilateral, $n=23$ bilateral) and overlap syndrome between Menière's disease and vestibular migraine $(n=5)$. There were no significant differences between the patients who completed the study period and the patients who dropped out, regarding age $(p=0.475)$, gender $(p=0.309)$ and vestibular diagnosis $(p=0.367)$.

\section{Response rates}

Figure 1 presents the average response rates of fully completed questionnaires of the DizzyQuest. The response rates were $90 \%$ for the morning-questionnaire, $62 \%$ for the withinday-questionnaire and $87 \%$ for the evening-questionnaire. The response rates of the morning, day and evening questionnaires were significantly different from the desired a priori defined response rate of $>50 \%(p<0.001)$ respectively.

\section{Examples of outcome measures of the DizzyQuest on an individual level}

The DizzyQuest enables the opportunity to give additional insights in fluctuation of symptoms during a specific time period (e.g., day or week), and to investigate 
Table 3 Characteristics of patients who dropped out during this study

\begin{tabular}{lllll}
\hline $\begin{array}{l}\text { Patient } \\
\text { number }\end{array}$ & Diagnosis & Gender & Age (years) & Reason for drop-out \\
\hline 1 & VM/PPPD & Female & 63 & Prioritized treatment for another medical disorder \\
2 & MD & Female & 43 & Could not combine the application with work \\
3 & MD & Female & 37 & Changed her mind regarding participation after inclusion in study \\
4 & BV & Female & 44 & Prioritized treatment for another medical disorder \\
5 & PPPD & Female & 51 & Technical problems: did not receive evening questionnaires after using another device \\
6 & MD & Female & 59 & Technical problems: incorrect login \\
7 & MD & Female & 52 & Technical problems: incorrect login \\
8 & MD & Male & 59 & Frequency of within-day questionnaires was too high to combine participation in this \\
& & & & study with personal life \\
9 & DFNA9 & Male & 70 & Technical problems: dissatisfied about the application \\
10 & MD & Female & 64 & Personal circumstances \\
11 & UVP & Female & 61 & Technical problems: application did not work properly \\
12 & MD & Female & 44 & Death of family member \\
\hline
\end{tabular}

$V M$ vestibular migraine, $P P P D$ persistent postural perceptual dizziness, $M D$ Menière's disease, $U V P$ unilateral vestibulopathy, $D F N A 9$ genetic type of cochleovestibular dysfunction, leading to deafness and bilateral vestibulopathy

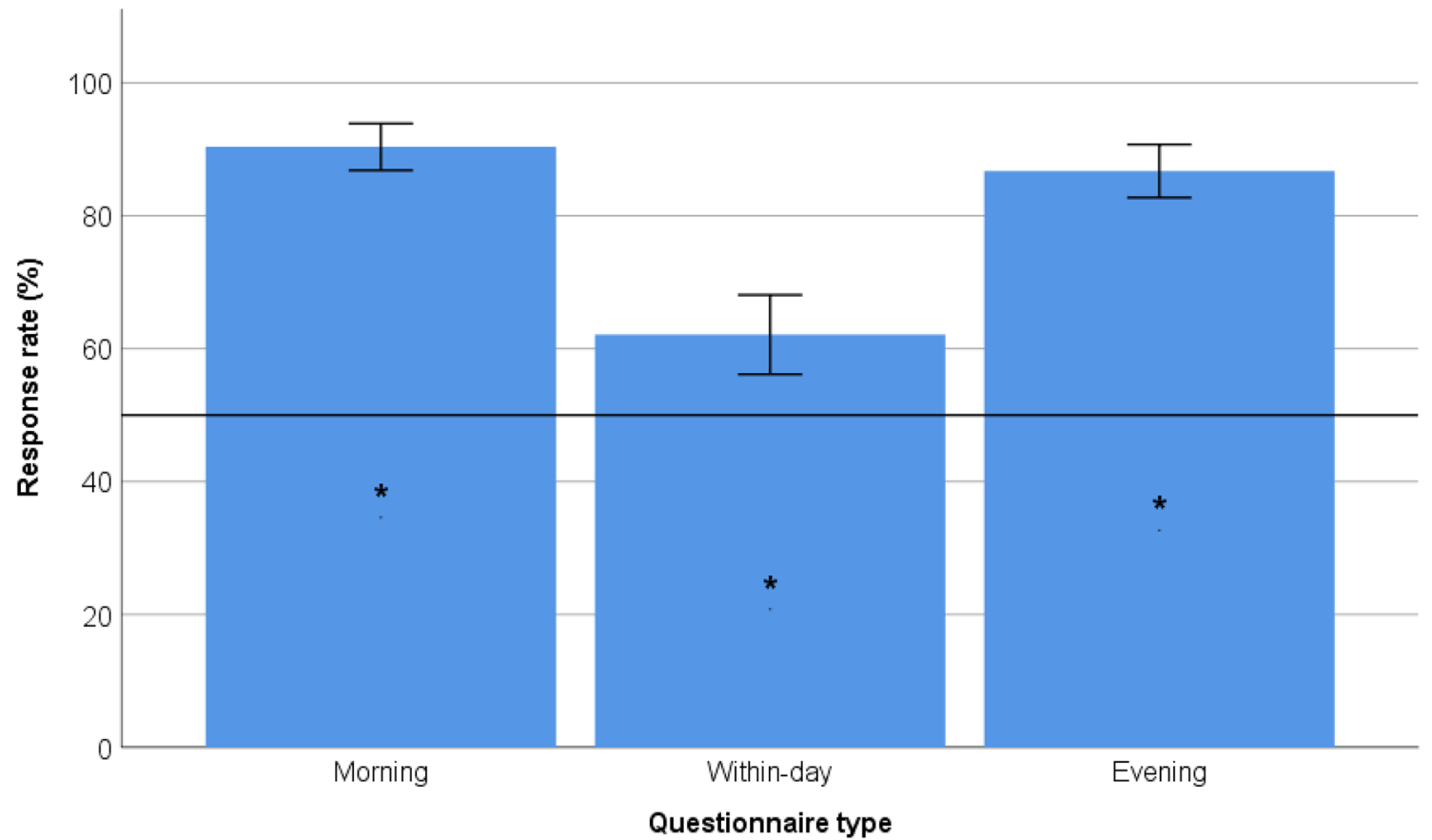

Fig. 1 The average response rates of fully completed questionnaires of the DizzyQuest. Error bars indicate 95\% confidence intervals of average response rates of all participants on the specific question-

the appearance of symptoms in relation to psychosocial context (Table 2). Figure 2a, b present examples of the association of imbalance and general well-being during a day (Fig. 2a) and a month (Fig. 2b) in a patient with Menière's disease, as a representative sample of results obtained in this study. Figure 3 presents the average scores of all 10 administered within-day-questionnaires during naire. The black horizontal bar indicates the desired response rate of $>50 \%$. Asterisks indicate a statistically significant difference in response rate from the desired $>50 \%$ response rate

six consecutive days regarding imbalance and general well-being in the same patient. These figures show that with the DizzyQuest, it is possible to calculate correlations between the appearance of symptoms and their context, on an individual and group level (see below "Appearance of neuro-otological symptoms in relation to psychosocial context"). 


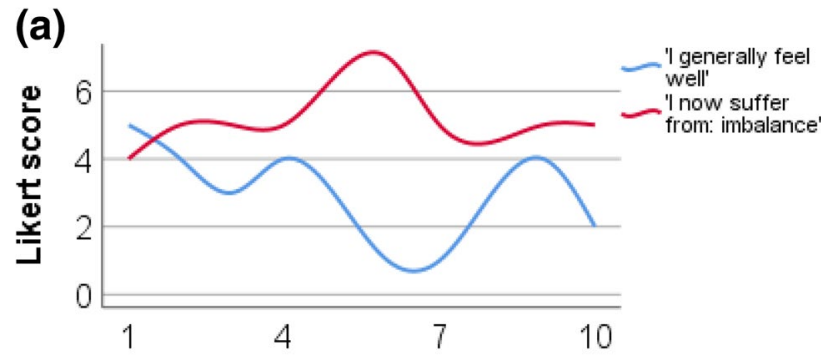

Number of questionnaire (day 5 )

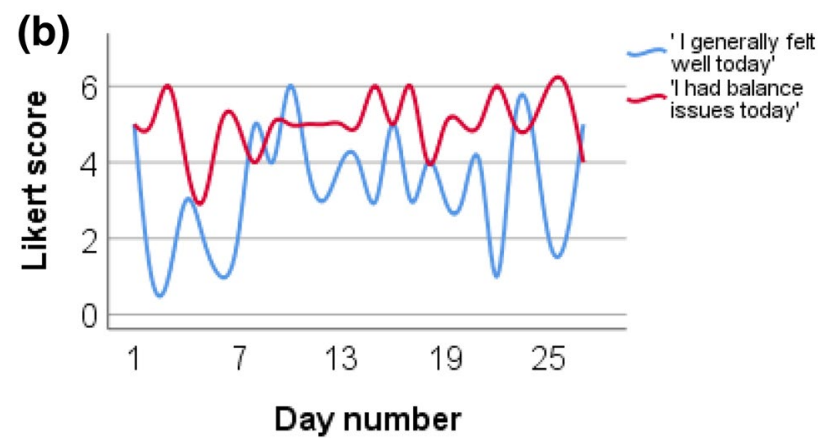

Fig. 2 a, b Examples of DizzyQuest results of a patient with Menière's disease regarding imbalance (red) and general well-being (blue) during one specific day and 1 month, as a representative sample of results obtained in this study. Likert scores on the y-axes vary from 1 (not at all) to 7 (very). a Illustrates the results of the 10 within-day-questionnaires that were administered on day five of the study. b Illustrates the results of the evening questionnaires obtained during 28 consecutive days

\section{Examples of outcome measures of the DizzyQuest on group level}

\section{Frequency of reported symptoms in the within-day-questionnaire (ESM method)}

In total, 51 patients reported 5829 neuro-otological symptoms in the within-day-questionnaire. Figure 4 demonstrates the mean reported frequency (\%) of neuro-otological symptoms, obtained with the within-day questionnaire, during six consecutive days in 51 patients. These are graphically displayed in three subgroups by diagnosis: Menière's disease $(n=19)$, bilateral vestibulopathy $(n=23)$, and other vestibular disorders $(n=9)$. Mostly reported symptoms were tinnitus and imbalance, while light flashes were least reported. Furthermore, variability can be observed between type of symptoms and subgroups of vestibular disorders. Patients with bilateral vestibulopathy reported imbalance significantly more often than patients with Menière's disease $(p=0.003, \mathrm{OR}=5.279,95 \% \mathrm{CI} 1.917 ; 14.533)$ and patients with other vestibular diagnoses $(p=0.012$, OR $=0.142$, $95 \%$ CI $0.037 ; 0.540$ ). Patients with bilateral vestibulopathy reported also significantly more often visual problems
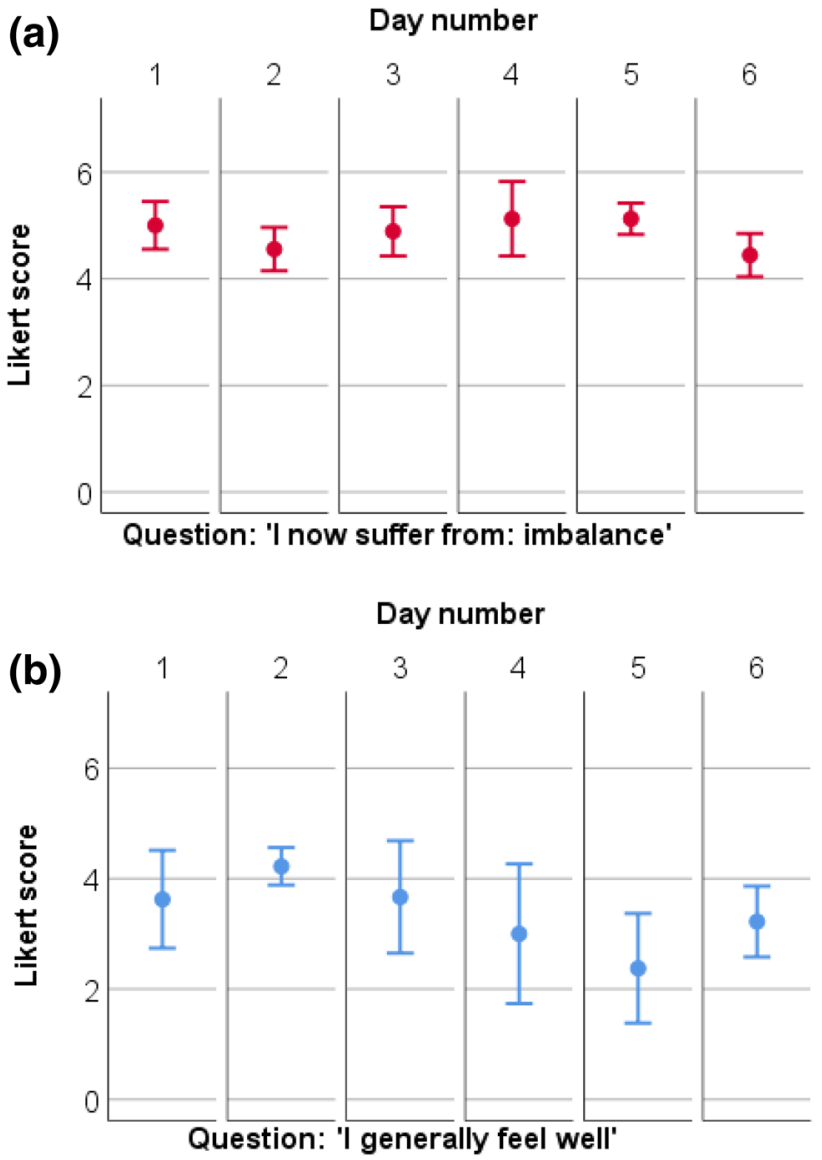

Fig. 3 a, b Average daily scores of imbalance (red, a) and general well-being (blue, b) obtained with the within-day-questionnaires that were administered 10 times a day during six consecutive days, in the same patient with Menière's disease. Dots represent the average scores of all completed within-day-questionnaires during a specific day. Error-bars show the 95\% confidence intervals

when moving compared to patients with Menière's disease $(p=0.033, \mathrm{OR}=7.735,95 \%$ CI $1.595 ; 37.524)$ and patients with other vestibular diagnoses $(p<0.001, \mathrm{OR}=0.038,95 \%$ CI $0.012 ; 0.126)$.

\section{Appearance of neuro-otological symptoms in relation to psychosocial context}

The appearance of three neuro-otological symptoms (imbalance, tinnitus and visual problems while moving), and their association with questions about positive affect ("I generally feel well at the moment") and negative affect ("I'm worrying about things") is presented in Table 4. Linear mixedmodel regression analysis showed that the appearance of imbalance, tinnitus and visual problems while moving, were nearly always significantly associated with a decrease in "I generally feel well at the moment" for all subgroups of vestibular disorders (except visual problems when moving in patients with Menière's disease). Regarding the question 


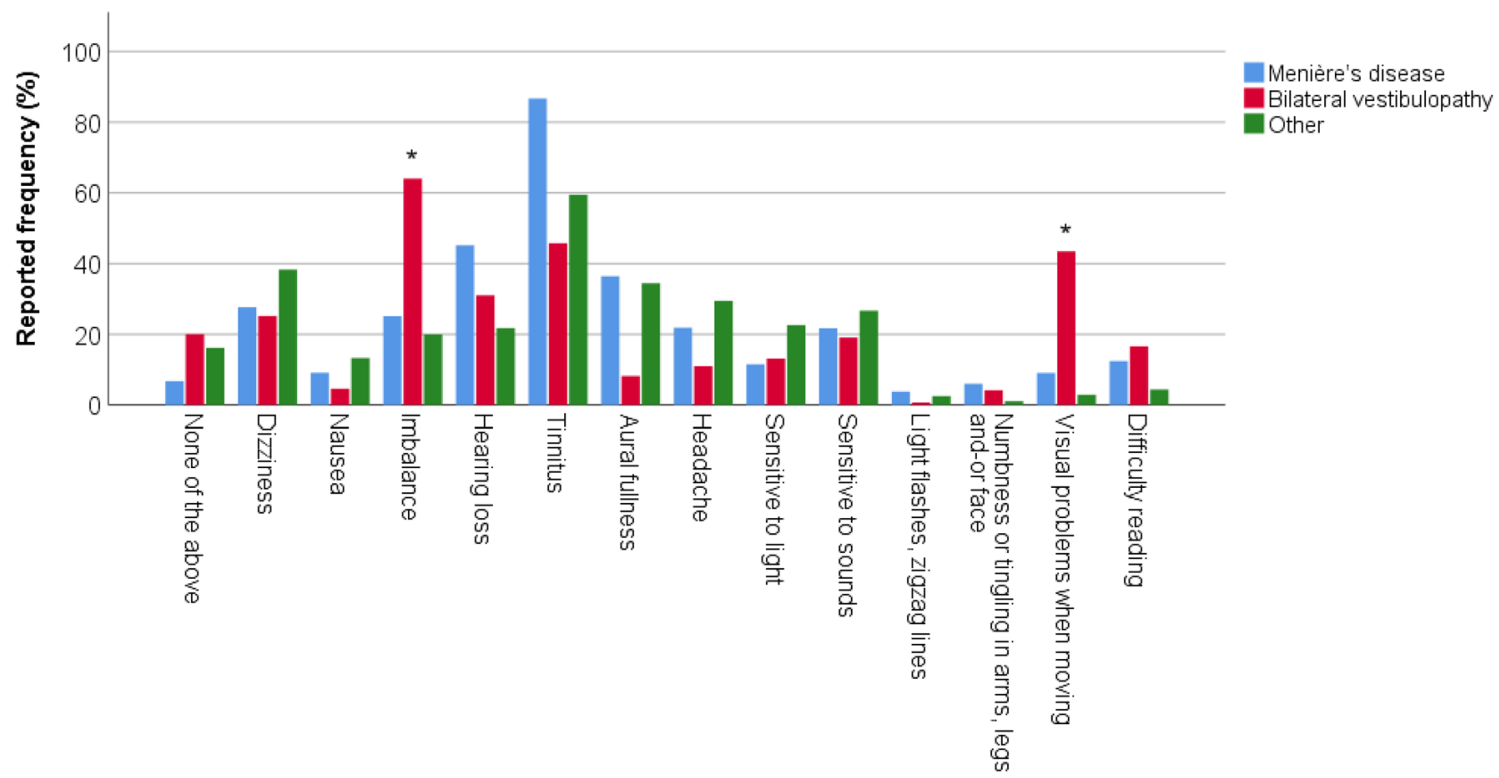

Symptoms

Fig. 4 Mean reported frequency (\%) of neuro-otological symptoms, obtained with the within-day questionnaire, during six consecutive days in 51 patients, divided in three subgroups of vestibular disorders.
Asterisks above a subgroup bar indicate a statistically significant difference in reported frequency of a symptom in that specific subgroup, compared to the other two
Table 4 Results of the mixedeffects linear regression analyses regarding the appearance of three neurootological symptoms and two questions about positive affect and negative affect, calculated for three subgroups of vestibular disorders

\begin{tabular}{|c|c|c|c|c|c|c|c|c|}
\hline \multirow[t]{4}{*}{ Symptoms } & \multicolumn{8}{|c|}{ Questions regarding positive affect and negative affect } \\
\hline & \multicolumn{4}{|c|}{ I generally feel well at the moment } & \multicolumn{4}{|c|}{ I'm worrying about things } \\
\hline & \multirow[t]{2}{*}{ Estimate } & \multicolumn{2}{|l|}{$95 \% \mathrm{CI}$} & \multirow[t]{2}{*}{$p$-value } & \multirow[t]{2}{*}{ Estimate } & \multicolumn{2}{|l|}{$95 \% \mathrm{CI}$} & \multirow[t]{2}{*}{$p$-value } \\
\hline & & Lower & Upper & & & Lower & Upper & \\
\hline Imbalance $=$ no & $-0.466^{\mathrm{a}}$ & -0.697 & -0.234 & $<0.001 *$ & $0.193^{\mathrm{a}}$ & 0.044 & 0.341 & $0.014 *$ \\
\hline Tinnitus $=$ no & $-0.413^{\mathrm{a}}$ & -0.670 & -0.155 & $0.003 *$ & $0.177^{\mathrm{a}}$ & -0.057 & 0.411 & 0.132 \\
\hline \multirow{3}{*}{$\begin{array}{l}\text { Visual problems } \\
\text { when mov- } \\
\text { ing = no }\end{array}$} & BV: -0.234 & -0.440 & 0.029 & $0.025^{*}$ & \multirow[t]{3}{*}{$0.12^{\mathrm{a}}$} & \multirow[t]{3}{*}{-0.046} & \multirow[t]{3}{*}{0.290} & \multirow[t]{3}{*}{0.156} \\
\hline & Other: -1.163 & -1.877 & -0.450 & $0.001 *$ & & & & \\
\hline & MD: -0.147 & -0.624 & 0.331 & 0.547 & & & & \\
\hline
\end{tabular}

$B V$ bilateral vestibulopathy, $M D$ Menière's disease

$* p<0.05$

${ }^{a}$ Applicable to all subgroups
"I'm worrying about things", the presence of imbalance was associated with a lower score.

\section{Frequency and nature of attacks of vertigo or dizziness}

The attack-questionnaire was used 159 times to indicate an attack of vertigo or dizziness. The median of number of reported vertigo attacks in the attack questionnaire per day was 0.071 for patients with Menière's disease, 0.036 for patients with bilateral vestibulopathy and 0.143 for patients with other vestibular disorders. Patients in the subgroup "other vestibular disorders" reported significantly more often a vertigo attack than patients in the subgroup "bilateral vestibulopathy" ( $p=0.033)$.

On an individual level, it was observed that the attackand evening-questionnaires showed different results regarding the reported frequency and nature (e.g., triggers, duration) of attacks of vertigo or dizziness. This interesting finding was outside the scope of this article, since it mainly results from the differences between reporting strategies: event sampling (attack-questionnaire) and time sampling (evening-questionnaire). This will therefore be discussed separately [52]. 


\section{Discussion}

The objective of this study was to introduce the first version of the DizzyQuest, to evaluate its response rates, and to provide examples of DizzyQuest outcome measures which can be used in future research. Response rates to all investigated questionnaires of the DizzyQuest were significantly higher than the desired response rate of $>50 \%$. The DizzyQuest is able to capture a variety of symptoms, including significant variability of symptomatology within and between participants and between subgroups of vestibular disorders. Furthermore, it was demonstrated that the Dizzyquest facilitates investigation of significant relations between symptoms and their psychosocial context. This is the first time that ESM has been used to assess symptoms in a population with vestibular disorders.

Main advantage of the DizzyQuest is the fact that it dynamically captures symptoms and their psychosocial context, by administering questionnaires multiple times to the same patient during patients' daily life. Therefore, it allows specific insights in the course of symptoms over time, and how they correlate with psychosocial factors. The DizzyQuest measures "in the moment" (within-dayquestionnaire) or with limited retrospection (morning-, evening- and attack-questionnaire), herewith decreasing recall bias and improving reliability. Since it is used in daily life, the ecological validity might be improved compared to many currently available questionnaires which are administered once a week at most. These aspects will likely be very valuable when investigating the effect of therapies for vestibular disorders, especially in cases of fluctuating symptoms like in Menière's disease [40, 53]. In the future, it could open doors to ecological momentary interventions, which take place in real time and in real world [54]. The repetitive measurement of the DizzyQuest also provides much larger numbers of data about the same topics, in and between patients. This allows for higher statistical accuracy in a smaller number of patients, and provides the opportunity of patients serving as their own control condition during intervention studies (e.g., $n=1$ study [55]). Moreover, symptom occurrence and worsening can be understood, since ESM allows the study of the mutual interaction of symptoms. Taking these considerations into account, the DizzyQuest could be used as a (complementary) tool for investigating symptoms and quality of life in patients with vestibular disorders.

Average response rates of the morning-questionnaire were the highest, while those of the within-day-questionnaire were the lowest. Additionally, the drop-out rate was $19 \%$ of patients. Fortunately, no patients dropped out due to any experienced psychological burden related to DizzyQuest use. Mostly reported reasons for drop-out involved technical problems and personal circumstances (e.g., combining the DizzyQuest with daily life activities). Regarding technical problems, these might be addressed by more strict monitoring of the patients in future trials, and to provide technical help if necessary. However, personal circumstances as a reason for drop-out, imply that responding to random signals is demanding in a practical way. Subjects have only $15 \mathrm{~min}$ to respond. Especially the within-day-questionnaire could be perceived as a burden, according to patients' feedback. After all, the within-day-questionnaire was administered 10 times a day in the first week. By providing questions 10 times a day, it was aimed to reach at least five completed withinday-questionnaires per day. Therefore, a lower response rate was expected compared to the morning and evening DizzyQuest questionnaires. Although the desired response rate was reached, the average response rate on the withinday-questionnaire in the patients that completed the whole study period, was slightly lower than in similar research across different domains [56, 57]. It is therefore strongly advised, especially when using the demanding within-dayquestionnaire, to provide patients with proper guidance during the study period. This might increase adherence [58]. Another complementary strategy would be to lower the within-day-questionnaire frequency, however, at the risk of getting too little data.

A variety of symptoms was reported within and between patients. It should be noted that these symptoms reflect the study population, and not the patients with vestibular disorders in general. For example, tinnitus was the most reported symptom, probably reflecting the overrepresentation of Menière's disease patients in this study population. Nevertheless, it has been shown that the DizzyQuest captured different profiles of symptomatology between subgroups of vestibular disorders. For example, imbalance and visual problems while moving (referring to oscillopsia) were significantly more reported in patients with bilateral vestibulopathy. These findings were expected, since they (partially) reflect the diagnostic criteria established for this disorder [46]. In addition, patients with bilateral vestibulopathy reported on average the lowest amount of vertigo attacks. Taking these results into account, it can be concluded that the DizzyQuest is able to measure different profiles of symptomatology between patients with different vestibular disorders.

Furthermore, the DizzyQuest facilitated the investigation of the relationship between neuro-otological symptoms and their psychosocial context. It was demonstrated that psychosocial well-being can be significantly negatively influenced by neuro-otological symptoms like imbalance, tinnitus and oscillopsia. This is congruent with previous literature in which it was shown that patients with vertigo or dizziness are more prone to have psychiatric co-morbidity and in these 
cases more often report depression, anxiety and vertigorelated handicap [59]. The DizzyQuest might therefore be used as a complementary tool to further explore the interface between neuro-otological symptoms and their psychosocial context in future research.

\section{Limitations}

A subset of the study population was loss-to-follow-up. This implies that results are only a reflection of the patients that completed the whole 4-week study period. Whether this has induced any selection bias, cannot be ruled out. Furthermore, completing the questionnaires might take some time, especially in the beginning. However, bothersome time consumption was not measured in this study. After familiarization with the questionnaires, time to complete each questionnaire dropped to on average $2 \mathrm{~min}$, according to the patients' feedback.

In this first article about the DizzyQuest, it would have been preferred to also report on its content and construct validity. However, since the development of the DizzyQuest was a very long and thorough process according to the COSMIN guidelines [60], it was decided to report this separately in an article fully devoted to the validity of the DizzyQuest (manuscript in preparation).

\section{Future steps to DizzyQuest}

To fully capture symptoms of patients and their psychosocial context, focus groups were recently conducted, in which the questionnaires were discussed in a standardized way [56, 61]. Patients and doctors got the opportunity to include, exclude and rephrase questionnaire items. These results will be implemented in the updated version of the DizzyQuest. Furthermore, the DizzyQuest should also be validated, including comparison to currently used questionnaires like the DHI and HADS (concurrent validity), and it should be translated into multiple languages. Preliminary observations showed in some cases discrepancies between reported symptoms in the within-day-questionnaire and the evening-questionnaire (data not shown). This is probably due to the different nature of the questionnaires (momentary versus retrospective design) and their timing (multiple times a day versus only one time during the evening).

Moreover, recent technological innovations resulted in portable hearing tests [62], video-oculography goggles for home use [63] and wearable movement sensors [64]. Simultaneous data-acquisition of these devices in combination with the DizzyQuest during daily life, might result in clinically useful insights regarding the correlation between subjective symptoms and objective measurements in patients with vestibular disorders. Finally, it should be noted that the frequency of administering the DizzyQuest questionnaires is not fixed. For example, in this study the within-day-questionnaire was administered 10 times a day for 1 week. However, this could be changed to e.g., 6 times a day for 2 weeks. Currently, two versions of the DizzyQuest are being developed for use in the near future: an updated extensive version for research including all questionnaires mentioned in this study, and a short DizzyQuest including only the evening- and attack-questionnaire. This latter should be able to serve as a standardized vestibular diary with relatively little retrospectivity, but without the burden of the within-dayquestionnaire. However, it should be noted that not using momentary assessments might decrease the sensitivity and the ability to investigate symptom appearance in relation to psychosocial context [65].

\section{Conclusion}

The DizzyQuest is able to capture vestibular symptoms within their psychosocial context in daily life, with little recall bias and high ecological validity. The DizzyQuest reached the desired response rates and showed different disease profiles between subgroups of patients with different vestibular disorders. This is the first time ESM was used to assess daily symptoms and quality of life in vestibular disorders, showing that it might be a useful tool in this population.

\section{Compliance with ethical standards}

Conflicts of interest All authors declare that they have no conflict of interest.

Ethics approval This study was in accordance with the legislation and ethical standards on human experimentation in the Netherlands and in accordance with the Declaration of Helsinki (amended version 2013). The medical ethical committee of Maastricht UMC+ approved this study (2018-0809).

Consent to participate/for publication Written informed consent was obtained from all patients, prior to participation.

Open Access This article is licensed under a Creative Commons Attribution 4.0 International License, which permits use, sharing, adaptation, distribution and reproduction in any medium or format, as long as you give appropriate credit to the original author(s) and the source, provide a link to the Creative Commons licence, and indicate if changes were made. The images or other third party material in this article are included in the article's Creative Commons licence, unless indicated otherwise in a credit line to the material. If material is not included in the article's Creative Commons licence and your intended use is not permitted by statutory regulation or exceeds the permitted use, you will need to obtain permission directly from the copyright holder. To view a copy of this licence, visit http://creativecommons.org/licenses/by/4.0/. 


\section{References}

1. Grill E, Heuberger M, Strobl R, Saglam M, Holle R, Linkohr B et al (2018) Prevalence, determinants, and consequences of vestibular hypofunction. Results from the KORA-FF4 survey. Front Neurol 9:1076

2. Gopinath B, McMahon CM, Rochtchina E, Mitchell P (2009) Dizziness and vertigo in an older population: the Blue Mountains prospective cross-sectional study. Clin Otolaryngol 34(6):552-556

3. Ward BK, Agrawal Y, Hoffman HJ, Carey JP, Della Santina CC (2013) Prevalence and impact of bilateral vestibular hypofunction: results from the 2008 US National Health Interview Survey. JAMA Otolaryngol Head Neck Surg. 139(8):803-810

4. Kim S, Oh YM, Koo JW, Kim JS (2011) Bilateral vestibulopathy: clinical characteristics and diagnostic criteria. Otol Neurotol. 32(5):812-817

5. Newman-Toker DE, Edlow JA (2015) TiTrATE: a novel, evidencebased approach to diagnosing acute dizziness and vertigo. Neurol Clin 33(3):577-599, viii

6. Lucieer F, Duijn S, Van Rompaey V, Pérez Fornos A, Guinand N, Guyot JP et al (2018) Full spectrum of reported symptoms of bilateral vestibulopathy needs further investigation-a systematic review. Front Neurol 9:352

7. Jacobson GP, Newman CW (1990) The development of the Dizziness Handicap Inventory. Arch Otolaryngol Head Neck Surg 116(4):424-427

8. Zigmond AS, Snaith RP (1983) The hospital anxiety and depression scale. Acta Psychiatr Scand 67(6):361-370

9. Ware JE Jr, Sherbourne CD (1992) The MOS 36-item short-form health survey (SF-36). I. Conceptual framework and item selection. Med Care. 30(6):473-483

10. Morgan MT, Friscia LA, Whitney SL, Furman JM, Sparto PJ (2013) Reliability and validity of the Falls Efficacy Scale-International (FES-I) in individuals with dizziness and imbalance. Otol Neurotol. 34(6):1104-1108

11. Myin-Germeys I, Oorschot M, Collip D, Lataster J, Delespaul P, van Os J (2009) Experience sampling research in psychopathology: opening the black box of daily life. Psychol Med 39(9):1533-1547

12. Shiffman S, Stone AA, Hufford MR (2008) Ecological momentary assessment. Ann Rev Clin Psychol 4:1-32

13. van Os J, Verhagen S, Marsman A, Peeters F, Bak M, Marcelis M et al (2017) The experience sampling method as an mHealth tool to support self-monitoring, self-insight, and personalized health care in clinical practice. Depress Anxiety 34(6):481-493

14. Yeo NL, White MP, Ronan N, Whinney DJ, Curnow A, Tyrrell J (2018) Stress and unusual events exacerbate symptoms in Meniere's disease: a longitudinal study. Otol Neurotol. 39(1):73-81

15. Goldberg RL, Piccirillo ML, Nicklaus J, Skillington A, Lenze E, Rodebaugh TL et al (2017) Evaluation of ecological momentary assessment for tinnitus severity. JAMA Otolaryngol Head Neck Surg. 143(7):700-706

16. Wenze SJ, Miller IW (2010) Use of ecological momentary assessment in mood disorders research. Clin Psychol Rev 30(6):794-804

17. Hartmann JA, Wichers M, Menne-Lothmann C, Kramer I, Viechtbauer W, Peeters F et al (2015) Experience sampling-based personalized feedback and positive affect: a randomized controlled trial in depressed patients. PLoS ONE. 10(6):e0128095

18. Moore RC, Depp CA, Wetherell JL, Lenze EJ (2016) Ecological momentary assessment versus standard assessment instruments for measuring mindfulness, depressed mood, and anxiety among older adults. J Psychiatr Res 75:116-123

19. Broen MP, Marsman VA, Kuijf ML, Van Oostenbrugge RJ, van Os J, Leentjens AF (2016) Unraveling the relationship between motor symptoms, affective states and contextual factors in Parkinson's disease: a feasibility study of the experience sampling method. PLoS ONE 11(3):e0151195

20. Patel M, Agarwal K, Arshad Q, Hariri M, Rea P, Seemungal $\mathrm{BM}$ et al (2016) Intratympanic methylprednisolone versus gentamicin in patients with unilateral Ménière's disease: a randomised, double-blind, comparative effectiveness trial. Lancet. 388(10061):2753-2762

21. Fornos AP, van de Berg R, Armand S, Cavuscens S, Ranieri M, Cretallaz C et al (2019) Cervical myogenic potentials and controlled postural responses elicited by a prototype vestibular implant. J Neurol 266(Suppl 1):33-41

22. Guinand N, Van de Berg R, Cavuscens S, Ranieri M, Schneider E, Lucieer F et al (2017) The video head impulse test to assess the efficacy of vestibular implants in humans. Front Neurol 8:600

23. Guinand N, Van de Berg R, Cavuscens S, Stokroos R, Ranieri $\mathrm{M}$, Pelizzone $\mathrm{M}$ et al (2016) Restoring visual acuity in dynamic conditions with a vestibular implant. Front Neurosci 10:577

24. Guinand N, van de Berg R, Cavuscens S, Stokroos RJ, Ranieri M, Pelizzone M et al (2015) Vestibular implants: 8 years of experience with electrical stimulation of the vestibular nerve in 11 patients with bilateral vestibular loss. ORL J Oto-rhino-laryngol Relat Spec 77(4):227-240

25. Guinand N, van de Berg R, Ranieri M, Cavuscens S, DiGiovanna J, Nguyen TA et al (2015) Vestibular implants: hope for improving the quality of life of patients with bilateral vestibular loss. Conf Proc IEEE Eng Med Biol Soc 2015:7192-7195

26. Guyot JP, Perez Fornos A, Guinand N, van de Berg R, Stokroos R, Kingma H (2016) Vestibular assistance systems: promises and challenges. J Neurol 263(Suppl 1):S30-S35

27. Nguyen TA, DiGiovanna J, Cavuscens S, Ranieri M, Guinand N, van de Berg R et al (2016) Characterization of pulse amplitude and pulse rate modulation for a human vestibular implant during acute electrical stimulation. J Neural Eng 13(4):046023

28. Nguyen TAK, Cavuscens S, Ranieri M, Schwarz K, Guinand N, van de Berg R et al (2017) Characterization of cochlear, vestibular and cochlear-vestibular electrically evoked compound action potentials in patients with a vestibulo-cochlear implant. Front Neurosci 11:645

29. Pelizzone M, Fornos AP, Guinand N, van de Berg R, Kos I, Stokroos R et al (2014) First functional rehabilitation via vestibular implants. Cochlear Implants Int 15(Suppl 1):S62-S64

30. Perez Fornos A, Cavuscens S, Ranieri M, van de Berg R, Stokroos $\mathrm{R}$, Kingma $\mathrm{H}$ et al (2017) The vestibular implant: a probe in orbit around the human balance system. J Vestib Res Equilib Orient 27(1):51-61

31. Perez Fornos A, Guinand N, van de Berg R, Stokroos R, Micera S, Kingma $\mathrm{H}$ et al (2014) Artificial balance: restoration of the vestibulo-ocular reflex in humans with a prototype vestibular neuroprosthesis. Front Neurol 5:66

32. Seppen BF, van Hoof M, Stultiens JJA, van den Boogert T, Guinand N, Guyot JP et al (2019) Drafting a surgical procedure using a computational anatomy driven approach for precise, robust, and safe vestibular neuroprosthesis placement-when one size does not fit all. Otol Neurotol. 40(5S Suppl 1):S51-S58

33. Starkov D, Guinand N, Lucieer F, Ranieri M, Cavuscens S, Pleshkov M et al (2020) Restoring the high-frequency dynamic visual acuity with a vestibular implant prototype in humans. Audiol Neuro-otol 25(1-2):91-95

34. Stultiens JJA, Postma AA, Guinand N, Perez Fornos A, Kingma $\mathrm{H}$, van de Berg R (2020) Vestibular implantation and the feasibility of fluoroscopy-guided electrode insertion. Otolaryngol Clin N Am 53(1):115-126

35. van de Berg R, Guinand N, Guyot JP, Kingma H, Stokroos RJ (2012) The modified ampullar approach for vestibular implant 
surgery: feasibility and its first application in a human with a longterm vestibular loss. Front Neurol 3:18

36. van de Berg R, Guinand N, Nguyen TA, Ranieri M, Cavuscens S, Guyot JP et al (2014) The vestibular implant: frequency-dependency of the electrically evoked vestibulo-ocular reflex in humans. Front Syst Neurosci 8:255

37. van de Berg R, Guinand N, Ranieri M, Cavuscens S, Khoa Nguyen TA, Guyot JP et al (2017) The vestibular implant input interacts with residual natural function. Front Neurol 8:644

38. van de Berg R, Guinand N, Stokroos RJ, Guyot JP, Kingma H (2011) The vestibular implant: quo vadis? Front Neurol. 2:47

39. van de Berg R, Lucieer F, Guinand N, van Tongeren J, George E, Guyot JP et al (2017) The vestibular implant: hearing preservation during intralabyrinthine electrode insertion-a case report. Front Neurol 8:137

40. Strupp M, Kraus L, Schautzer F, Rujescu D (2018) Meniere's disease: combined pharmacotherapy with betahistine and the MAO-B inhibitor selegiline-an observational study. J Neurol 265(Suppl 1):80-85

41. Zwergal A, Brandt T, Magnusson M, Kennard C (2016) DIZZYNET: the European network for vertigo and balance research. J Neurol 263(Suppl 1):S1

42. Zwergal A, Grill E, Lopez C, Dieterich M (2019) DIZZYNET 2019: approaching the future of vestibular research. J Neurol 266(Suppl 1):1-2

43. Lempert T, Olesen J, Furman J, Waterston J, Seemungal B, Carey $\mathrm{J}$ et al (2013) Vestibular migraine: diagnostic criteria: consensus document of the Barany Society and the International Headache Society. Der Nervenarzt. 84(4):511-516

44. Lopez-Escamez JA, Carey J, Chung WH, Goebel JA, Magnusson M, Mandala M et al (2016) Diagnostic criteria for Meniere's disease. Consensus document of the Barany Society, the Japan Society for Equilibrium Research, the European Academy of Otology and Neurotology (EAONO), the American Academy of Otolaryngology-Head and Neck Surgery (AAO-HNS) and the Korean Balance Society. Acta Otorrinolaringologica Espanola. 67(1):1-7

45. Staab JP, Eckhardt-Henn A, Horii A, Jacob R, Strupp M, Brandt T et al (2017) Diagnostic criteria for persistent postural-perceptual dizziness (PPPD): Consensus document of the committee for the Classification of Vestibular Disorders of the Barany Society. J Vestib Res Equilib Orient 27(4):191-208

46. Strupp M, Kim JS, Murofushi T, Straumann D, Jen JC, Rosengren SM et al (2017) Bilateral vestibulopathy: diagnostic criteria Consensus document of the Classification Committee of the Barany Society. J Vestib Res Equilib Orient 27(4):177-189

47. Mujagic Z, Leue C, Vork L, Lousberg R, Jonkers DM, Keszthelyi D et al (2015) The Experience Sampling Method-a new digital tool for momentary symptom assessment in IBS: an exploratory study. Neurogastroenterol Motil 27(9):1295-1302

48. Bisdorff A, Von Brevern M, Lempert T, Newman-Toker DE (2009) Classification of vestibular symptoms: towards an international classification of vestibular disorders. J Vestib Res Equilib Orient 19(1-2):1-13

49. Engelen L, Held F (2019) Understanding the office: using ecological momentary assessment to measure activities, posture, social interactions, mood, and work performance at the workplace. Buildings. 9:54

50. Hanssen E, Balvert S, Oorschot M, Borkelmans K, van Os J, Delespaul P et al (2020) An ecological momentary intervention incorporating personalised feedback to improve symptoms and social functioning in schizophrenia spectrum disorders. Psychiatry Res 284:112695

51. Courvoisier DS, Eid M, Lischetzke T (2012) Compliance to a cell phone-based ecological momentary assessment study: the effect of time and personality characteristics. Psychol Assess 24(3):713-720

52. De Joode LEGH, Martin EC, Stultiens JJ, Leue C, Delespaul P, Peeters F et al (2020) The dizzyquest: to have or not to have... a vertigo attack? J Neurol. https://doi.org/10.1007/s00415-02010043-x

53. Sajjadi H, Paparella MM (2008) Meniere's disease. Lancet 372(9636):406-414

54. Heron KE, Smyth JM (2010) Ecological momentary interventions: incorporating mobile technology into psychosocial and health behaviour treatments. Br J Health Psychol 15(Pt 1):1-39

55. van der Velden RMJ, Mulders AEP, Drukker M, Kuijf ML, Leentjens AFG (2018) Network analysis of symptoms in a Parkinson patient using experience sampling data: an $n=1$ study. Mov Disord 33(12):1938-1944

56. Vork L, Keszthelyi D, Mujagic Z, Kruimel JW, Leue C, Ponten I et al (2018) Development, content validity, and cross-cultural adaptation of a patient-reported outcome measure for real-time symptom assessment in irritable bowel syndrome. Neurogastroenterol Motil 30(3):e13244

57. Berkel NV, Ferreira D, Kostakos V (2017) The experience sampling method on mobile devices. ACM Comput Surv. 50(6):1-40

58. Verhagen SJW, Berben JA, Leue C, Marsman A, Delespaul PAEG, van Os J et al (2017) Demonstrating the reliability of transdiagnostic mHealth Routine Outcome Monitoring in mental health services using experience sampling technology. PLoS ONE. 12(10): $\mathrm{e} 0186294$

59. Lahmann C, Henningsen P, Brandt T, Strupp M, Jahn K, Dieterich $\mathrm{M}$ et al (2015) Psychiatric comorbidity and psychosocial impairment among patients with vertigo and dizziness. J Neurol Neurosurg Psychiatry 86(3):302-308

60. Prinsen CAC, Vohra S, Rose MR, Boers M, Tugwell P, Clarke M et al (2016) How to select outcome measurement instruments for outcomes included in a "Core Outcome Set"-a practical guideline. Trials. 17(1):449

61. Herrewegh A, Vork L, Eurelings E, Leue C, Kruimel J, Van Koeveringe $\mathrm{G}$ et al (2018) The development of a patient-reported outcome measure for real-time symptom assessment in a population with functional urologic complaints-a focus group study. Neurourol Urodyn. 37:2893-2903

62. Tse D, Ramsay T, Lelli DA (2019) Novel use of portable audiometry to track hearing fluctuations in Meniere's disease: a pilot study. Otol Neurotol. 40(2):e130-e134

63. Young AS, Lechner C, Bradshaw AP, MacDougall HG, Black DA, Halmagyi GM et al (2019) Capturing acute vertigo: a vestibular event monitor. Neurology. 92(24):e2743-e2753

64. van der Weegen S, Essers H, Spreeuwenberg M, Verwey R, Tange $\mathrm{H}$, de Witte L et al (2015) Concurrent validity of the MOX activity monitor compared to the ActiGraph GT3X. Telemed J E-health 21(4):259-266

65. Schuster R, Kaiser T, Schreyer M, Berger T, Klein J, Moritz S, et al (2020) Effects of intense assessment on statistical power in randomized controlled trials: Informed simulation study on depression 


\section{Affiliations}

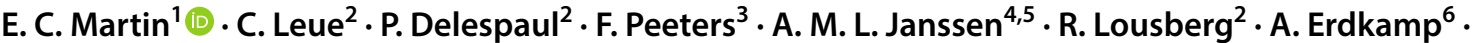 S. van de Weijer ${ }^{6}$. J. Widdershoven ${ }^{1} \cdot$ H. Blom ${ }^{7} \cdot$ T. Bruintjes $^{8} \cdot$ A. Zwergal ${ }^{9} \cdot$ E. Grill $^{10}$ - N. Guinand ${ }^{11}$. A. Perez-Fornos ${ }^{11} \cdot$ M. R. van de Berg ${ }^{1} \cdot$ J. J. A. Stultiens ${ }^{1} \cdot$ H. Kingma ${ }^{1,12} \cdot$ R. van de Berg ${ }^{1,12}$}

1 Division of Balance Disorders, Department of Otorhinolaryngology and Head and Neck Surgery, Maastricht University Medical Center+, Maastricht, The Netherlands

2 Department of Psychiatry and Neuropsychology, School for Mental Health and Neuroscience, Maastricht University Medical Center+, Maastricht, The Netherlands

3 Department of Clinical Psychological Science, Faculty of Psychology and Neuroscience, Maastricht University, Maastricht, The Netherlands

4 Department of ENT/Audiology, School for Mental Health and Neuroscience (MHENS), Maastricht University Medical Centre, Maastricht, The Netherlands

5 Department of Methodology and Statistics, Care and Public Health Research Institute (CAPHRI), Maastricht University, Maastricht, The Netherlands

6 Department of Psychiatry and Psychology, Maastricht University Medical Center, Maastricht, The Netherlands
7 Department of ENT, HagaZiekenhuis, The Hague, The Netherlands

8 Apeldoorns duizeligheidscentrum, Gelre ziekenhuizen, Apeldoorn, The Netherlands

9 Department of Neurology, Ludwig-Maximilians-University of Munich, Munich, Germany

10 Department of Medical Informatics, Ludwig-Maximilians-University of Munich, Munich, Germany

11 Service of Otorhinolaryngology Head and Neck Surgery, Department of Clinical Neurosciences, Geneva University Hospitals, Geneva, Switzerland

12 Faculty of Physics, Tomsk State Research University, Tomsk, Russia 